\title{
An assessment of high touch object cleaning thoroughness using a fluorescent marker in two Australian hospitals
}

\author{
Cathryn L. Murphy ${ }^{1,2,3,5}$ RN, PhD, MCNA, CICP, CIC \\ Deborough A. Macbeth ${ }^{1} \mathrm{RN}, \mathrm{PhD}, \mathrm{CICP}$ \\ Petra Derrington ${ }^{1} \mathrm{MBChB}, \mathrm{MSC}, \mathrm{MRCP}, \mathrm{MRCPath}, \mathrm{FRCPA}$ \\ John Gerrard ${ }^{1}$ RN, MBBS, MSC., FRACP \\ Jacinta Faloon ${ }^{1} \mathrm{RCpN} B \mathrm{HSC}$ \\ Kellie Kenway ${ }^{1} \mathrm{RN}, \mathrm{BN}, \mathrm{M}$ Adv, Prac, Infection, Prevention, CICP \\ Samantha Lavender ${ }^{1} \mathrm{RN}, \mathrm{BN}$ \\ Simon Leonard' RN, BN \\ Amanda Orr \\ Dayani Tobin ${ }^{1} \mathrm{RN}$ \\ Philip Carling ${ }^{4} \mathrm{MD}$ \\ ${ }^{1}$ Infection Control, Infectious Diseases and Microbiology Departments, Gold Coast Hospital, 108 Nerang Street, \\ Southport, Qld 4215, Australia. \\ ${ }^{2}$ School of Nursing and Midwifery, Griffith University, Southport, Qld 4215, Australia. \\ ${ }^{3}$ Faculty of Health Sciences and Medicine, Bond University, Qld 4229, Australia. \\ ${ }^{4}$ Hospital Epidemiology, Carney Hospital, 2100 Dorchester Avenue, Boston, MA 02124, USA. \\ ${ }^{5}$ Corresponding author. Email: Cathryn_Murphy@health.qld.gov.au
}

\begin{abstract}
Objective: We needed to better understand the usefulness of different methods of monitoring and achieving sustained improvement in cleaning. Common elements of successful international approaches included a covert nature, the use of a method to visually identify and highlight deficiencies in the cleaning of high touch objects (HTOs) and the provision of feedback and education before re-evaluation. The specific purpose of this study was to evaluate fluorescent marking, education and feedback for assessing and improving HTO cleaning in a typical Australian inpatient hospital setting.

Methods: A three-phase, prospective study was conducted in two acute care hospitals over 17 weeks. For each phase, in a set of 37 specific single-inpatient rooms, seven predefined HTOs were marked with a liquid isopropyl alcohol and optical brightener formulation targeting material solution containing a fluorescent marker (FM), known as $\mathrm{DAZO}^{\circledR}$, designed specifically for the purpose of evaluating surface cleaning. In each hospital we targeted rooms located in the four wards with the greatest de novo multidrug resistant organism burden. Forty-eight hours after applying the FM we used a black-light to indicate the mark's presence or removal. In phase 1 only, HTOs were swabbed before marking. Also only in the first phase and immediately following initial assessment, each HTO was cleaned, remarked and re-assessed at $48 \mathrm{~h}$. Between phases 1 and 2, investigators provided results to environmental services staff (EVS) leadership and staff after each phase. Education was provided to EVS staff after phase 1 only.

Results: A total of 986 marks were evaluated. The cleaning scores for individual HTOs in phases 1-3 ranged from 9.4 to $77.8 \%, 10.8$ to $93 \%$ and 13.5 to $67.7 \%$ respectively. In phase 3, three HTOs scored lower than their phase 1 level. The mean overall cleaning scores for phases $1-3$ were $34 \%, 53 \%$ and $41 \%$ respectively.

Conclusions: The FM was useful to assess HTO cleaning thoroughness. It facilitated relevant feedback and education and motivated staff to strive for continual improvements in environmental cleaning. Without on-going education, preliminary improvements were unsustained. However, investigators better understood flaws in cleaning and policy/procedure conflicts.
\end{abstract}

Received 16 September 2011, accepted 19 December 2011, published online dd mmm yyyy 


\section{Introduction}

A rapidly evolving understanding of healthcare-associated infections (HAIs) has lead to increased appreciation of the surface contamination of objects in patients' surroundings and their implication in the transmission of environmentally resilient pathogens such as Clostridium difficile, vancomycinresistant enterococci, methicillin-resistant Staphylococcus aureus, Acinetobacter baumannii and noroviruses. ${ }^{1-19}$ Each of these organisms are problematic in Australian hospitals and some clinicians have realised that their prevention requires thorough cleaning. ${ }^{20-24}$ However recent national infection control guidelines and priority areas under-emphasise the importance of enhanced monitoring of cleaning, better understanding of how it is routinely performed, and how it can and should be improved in Australian hospitals. ${ }^{25}$ Instead, infection control staff are compelled to invest substantial resources in collecting, analysing and submitting data relating to hand hygiene, Staphylococcal bacteraemia, and other state and nationally imposed priority areas. ${ }^{26,27}$ These regulatory requirements divert scarce infection control resources from locally important issues such as monitoring and improving thoroughness of environmental cleaning.

There are several differences between the structures and functions that respectively underpin United States (US) and Australian infection prevention programs which we believe currently impede improvements in hospital cleaning in Australia. For example, where they exist, cleaning directives from Australian state and territory authorities vary and no single agency is recognised as being the ultimate authority. Agencies that accredit Australian hospitals do not routinely require information about the effectiveness of cleaning. Published local, comprehensive research addressing and discussing the thoroughness of cleaning in Australian hospitals is scant. ${ }^{28}$ These gaps are especially alarming given recent serious microbiological events such as the detection of Australia's first cases of hypervirulent $C$. difficile ribotype 027 and the on-going problem of multiple resistant organisms. ${ }^{19,23,24,29,30}$ Australian experts are increasingly focussing on introducing more powerful chemical formulations for routine cleaning rather than evaluating and understanding the thoroughness of cleaning environmental surfaces. ${ }^{21}$

Internationally, opportunities to improve cleaning of patient rooms and high touch objects (HTOs) have been realised. ${ }^{4,5,8,10-12,15,31,32}$ US, Canadian and European researchers have recently reported success with the use of fluorescent target monitoring on HTOs and concurrent enhanced education of environmental services staff (EVS) staff. ${ }^{33-36}$

Located in southeast Queensland and part of the Gold Coast Health Service District (GCHSD), Hospitals A and B are publically funded, teaching hospitals with a combined total of $\sim 780$ available acute care inpatient beds. On average each month between 8000-8500 admissions are made to these facilities. Infection control procedures, EVS staff orientation, on-going education and cleaning supplies are standardised across both facilities. EVS staff in both hospitals are employees of the GCHSD and are provided with training at commencement of their job and then on an annual basis. At the time of this study internal quality improvement processes related to cleaning in both hospitals were limited to visual inspections by senior EVS staff. Subsequently, each hospital is working with the state Health Department to embrace recent standardised, state EVS initiatives including comprehensive auditing using a standardised audit tool. Our infection control and prevention team has substantial peer and professional relationships with leading global and US infection prevention researchers. We are aggressively pursuing excellence in every element of our program and consider research, innovation and collaboration to be important in this pursuit. We embraced a unique opportunity to use fluorescent marking to determine the extent to which a set of seven predetermined HTOs were routinely cleaned. We also investigated the impact of feeding back results and providing enhanced education to improve environmental cleaning. During the first phase of the study we cultured the surfaces of HTOs in our cohort of 37 rooms to use visual images of cultured pathogenic organisms to confront and educate EVS staff on the likelihood of contamination in their environments. After phase 1, we surveyed EVS staff regarding knowledge and cleaning, however detailed reporting and discussion of both the environmental culturing and the EVS survey results are beyond the scope of this report, as is any consideration of the correlation between thoroughness of cleaning results and infection rates in the hospitals.

\section{Methods}

\section{Advice to EVS}

Prior to the project and after reviewing the recommended cleaning frequencies for each of the HTOs we met with EVS management from both hospitals and provided non-specific advice about our intention to begin working collaboratively with them to better monitor, understand and improve cleaning across the District. We shared stories of international success with the fluorescent marker (FM), known as DAZO (Ecolab, St Paul, MN, USA), and our desire to implement a similar system including periodic provision of feedback and education. No information about specific HTOs, wards, rooms or time periods was exchanged.

\section{Ward, room and HTO selection}

We analysed the data of 2010 de novo multidrug resistant organisms (MDROs) to identify those wards in each hospital at or above the 75 th percentile. Four wards in each hospital met the inclusion criteria. At Hospital A we included every single inpatient room on each of those four wards and at Hospital B we selected a convenience sample of single inpatient rooms in the four target wards equal to the number in Hospital A. We studied the same HTOs from the same rooms and adjoining en suites, where they existed, in each phase of the study. Patient rooms and bathrooms were included regardless of whether they were occupied or empty; HTOs in bathrooms 
were excluded in the event that the bathroom was occupied at the time of either application or reading of the FM mark. Seven standardised HTOs (bedroom light switch, inside bedroom door handle, bedroom soap dispenser, toilet grab rail, toilet flush button, bedroom tap handle and bedroom paper towel dispenser) were selected following reviews of the literature and inspection of the objects routinely and permanently fixed in an inpatient room and adjoining bathroom at both hospitals. The three-phase, prospective study was conducted simultaneously in the two acute care hospitals over 17 weeks. A common rationale of developing a more comprehensive and detailed understanding of local cleaning practices for the purpose of designing locally appropriate educational material and targeted interventions underpinned phases $1-3$. The use of the FM for assessing cleaning thoroughness and the provision of results from the previous assessment where they existed (phases 2 and 3) were common interventions in each phase. Intervention unique to Phase 1 included:

- Collection and culturing of swabs from each HTO so as to have examples of serious, local pathogens to use for teaching purposes;

- Repeat application at $48 \mathrm{~h}$ and reading of the second markings a further $48 \mathrm{~h}$ later;

- Provision of education to EVS staff and survey of EVS staff to determine their existing levels of training and knowledge of frequency of HTO cleaning.

The aims of each phase were identical - to determine the thoroughness of specific HTO cleaning and to use findings to inform and incentivise EVS leadership and staff to improve HTO cleaning overall.

During the first phase of the study and before the application of the FM, the seven high touch areas were cultured by the principal investigators $(\mathrm{CM} \& \mathrm{DB})$ in the cohort of 37 rooms. Both investigators had been trained in the collection of environmental swabs. A plastic template measuring $30 \times 30 \mathrm{~mm}$ to give a total area of $900 \mathrm{~mm}^{2}$ was placed in an identical position for each HTO in each room. The inside area of this template was swabbed using a sterile swab (Interpath Copan sterile swabs in Amies Agar gel without charcoal; COPAN, Brescia, Italy). This template was cleaned after each sampling with an alcohol-free multisurface detergent wipe (Tuffie Wipes, Bolton, UK) to avoid cross contamination of HTOs or the template.

The swabs were plated out on horse blood agar and total aerobic colony counts were determined after $48 \mathrm{~h}$ of incubation at $37^{\circ}$ in air. Identification to species level was performed on all $S$. aureus and susceptibility testing was performed on Enterococcus spp. and $S$. aureus only in accordance with Clinical and Laboratory Standards Institute guidelines. $^{37}$

In each of the three phases, the principal investigators who had both been specifically trained in the use of the FM, marked and read the HTOs. Forty-eight hours after application, each investigator used a black light to determine the presence or removal of each placed mark. In phase 1 only, immediately following initial assessment, each HTO was cleaned, remarked and re-assessed after $48 \mathrm{~h}$. Once all marked targets in the room were evaluated, investigators removed all residual marks using a detergent wipe and black light to ensure all surfaces were clean and FM-free. Between phases 1 and 2, investigators administered a short written survey regarding current knowledge and training experience to EVS staff for self-completion. Immediately following the survey, the baseline results of cleaning assessment were provided along with standard face-to-face education to EVS staff at both hospitals. Education was not provided between phases 2 and 3.

At the time of marking and assessing each room, a work card was completed, noting the investigators' name, ward, bed number, date, time, the unique record number of the occupant, and which specific objects were marked, read and cleaned.

No patients or patient specimens were involved in this study. The unique record number of each patient accommodated in the room at the time of marking was noted to identify rooms accommodating patients known to be colonised/infected with MDROs. Investigators agreed to deidentify individual wards, limiting reference to them by type (e.g. surgical ward or medical ward) in order to protect the identities of the designated EVS staff responsible for cleaning those specific areas. The methodology used was proven for testing the efficacy of cleaning and therefore the project constituted a normal operational or quality activity. As such, ethics approval was not required. The project proposal was endorsed by the District Infection Control Committee in December 2010.

\section{Data and statistical analysis}

During every phase and in both hospitals, data collection and analysis was standardised. The two-tailed Fisher's exact test and $95 \%$ confidence intervals (CI) were performed using GraphPad InStat version 3.10 32-bit for Windows 95 (GraphPad Software Inc., San Diego, CA, USA). A $P$-value of $<0.05$ was considered significant.

\section{Results}

A total of 986 HTOs from 37 patient rooms were marked and evaluated in the study's three phases. Table 1 details the overall and hospital-specific aggregate proportion of cleaned HTOs by phase. At baseline, 34\% (95\% CI 30.1-38.4) of HTOs were cleaned. There was a statistically significant difference $(P=0.0367)$ between the overall baseline HTO rates at hospitals $\mathrm{A}$ and $\mathrm{B}$, which were $29.3 \%(95 \% \mathrm{CI}$ 23.7-35.4) and 38.3\% (95\% CI 28.4-44.5) respectively.

In phase 2 the overall cleaning rate increased significantly $(P \leq 0.0001)$ to $53.5 \%$ (95\% CI 47.2-59.6). Although both hospitals improved their overall proportion of cleaned HTOs, Hospital B (60.3\%; 95\% CI 51.8-68.3) again showed significantly better $(P=0.0289)$ cleaning results than Hospital A (45.6\%; 95\% CI 36.8-54.8). 
Table 1. Overall and hospital specific aggregate proportion of cleaned HTOs by phase

\begin{tabular}{lrccc}
\hline & $\begin{array}{c}\text { Objects } \\
\text { Cleaned }\end{array}$ & $\begin{array}{c}\text { Objects } \\
\text { Not } \\
\text { Cleaned }\end{array}$ & $\begin{array}{c}\text { Total No. } \\
\text { of Objects } \\
\text { Marked }\end{array}$ & $\begin{array}{c}\% \\
\text { Cleaned }\end{array}$ \\
\hline Phase 1 - 17/1/11-21/1/11 & 169 & 326 & 495 & $34.1 \%$ \\
Hospital A & 67 & 162 & 229 & $29.3 \%$ \\
Hospital B & 102 & 164 & 266 & $38.3 \%$ \\
Phase 2-14/3/11-16/3/11 & 131 & 114 & 245 & $53.5 \%$ \\
Hospital A & 52 & 62 & 114 & $45.6 \%$ \\
Hospital B & 79 & 52 & 131 & $60.3 \%$ \\
Phase 3-9/5/11-11/5/11 & 101 & 145 & 246 & $41.1 \%$ \\
Hospital A & 24 & 90 & 114 & $21.1 \%$ \\
Hospital B & 77 & 55 & 132 & $58.3 \%$ \\
Overall & 401 & 585 & 986 & $40.7 \%$ \\
\hline
\end{tabular}

In the final phase of the project the overall proportion of cleaned HTOs was 41\% (95\% CI 35.1-47.3). Overall thoroughness of cleaning dropped in both hospitals. Hospital A experienced a statistically significant reduction in cleaning from $46 \%$ (CI $9536.8-54.8$ ) in phase 2 compared with $21 \%(95 \%$ CI $14.6-29.4)$ in phase $3(P=0.0001)$. Hospital B's cleaning results were lower in phase $3(58.3 \%$; 95\% CI 49.8-66.4) compared with phase $2(60.3 \%$; 95\% CI 51.7-68.3) however the reduction was not significant. A highly significant $(P \leq 0.0001)$ difference between Hospital B and Hospital A's results was observed again in phase 3 .
Table 2 details the aggregate proportion of cleaning thorughness per specific HTO in each phase. Cleaning scores in phases 1-3 for individual HTOs ranged from 9.4 to $77.8 \%$, 10.8 to $93 \%$ and 13.5 to $67.7 \%$ respectively. In phase 3 , the two HTOs located in the bathrooms (grab bar and flush button) and the patient room tap handle were cleaned less often than at baseline. Aggregate results for each of the project's three phases indicate that the least and most frequently cleaned HTOs in the District were bedroom light switches $(10.8 \%$; 95\% CI 6.7-16.9) and grab bars (79.0\%; 95\% CI 71.0-85.3) respectively.

\section{Discussion}

Our study assesses the use of fluorescent targeting to evaluate hospital cleaning. It has the potential to raise awareness and appreciation among Australian and South East Asian clinicians, EVS, and other staff of the need to better understand and address thoroughness of cleaning as one element of local infection prevention programs.

Prior to undertaking this work we had no valid or reliable understanding of how thoroughly HTOs in our facilities were cleaned. After using this target marking solution we are well aware that even with intensive education, in one of our hospitals the thoroughness of HTO cleaning remained below 50\%. The FM assisted us to quantify and justify reprioritisation of competing infection control goals and redistribution of scarce infection control resources to focus on and strive for improved cleaning thoroughness. It has

Table 2. Aggregate proportion of cleaning thoroughness of specific HTOs by phase

\begin{tabular}{|c|c|c|c|c|c|c|c|}
\hline & Cleaned & Marked & $\%$ & Lower $95 \% \mathrm{CI}$ & Upper $95 \%$ & Plus & Minus \\
\hline & & & pitals & hase 1 & & & \\
\hline Bedroom Light Switch & 7 & 74 & 9.46 & 4.66 & 18.26 & 8.80 & 4.80 \\
\hline Door Handle & 26 & 74 & 35.14 & 25.24 & 46.5 & 11.36 & 9.90 \\
\hline Soap Dispenser & 7 & 74 & 9.46 & 4.66 & 18.26 & 8.80 & 4.80 \\
\hline Grab Bar & 49 & 63 & 77.78 & 66.09 & 86.27 & 8.49 & 11.69 \\
\hline Flush Button & 40 & 62 & 64.52 & 52.08 & 75.26 & 10.74 & 12.44 \\
\hline Tap Handle & 33 & 74 & 44.59 & 33.82 & 55.91 & 11.32 & 10.77 \\
\hline Towel Dispenser & 7 & 74 & 9.46 & 4.66 & 18.26 & 8.80 & 4.80 \\
\hline Bedroom Light Switch & 4 & 37 & 10.81 & 4.29 & 24.71 & 13.90 & 6.52 \\
\hline Door Handle & 20 & 37 & 54.05 & 38.38 & 68.96 & 14.91 & 15.67 \\
\hline Soap Dispenser & 11 & 37 & 29.73 & 17.49 & 45.78 & 16.05 & 12.24 \\
\hline Grab Bar & 28 & 30 & 93.33 & 78.68 & 98.15 & 4.82 & 14.65 \\
\hline Flush Button & 26 & 31 & 83.87 & 67.37 & 92.91 & 9.04 & 16.50 \\
\hline Tap Handle & 23 & 36 & 63.89 & 47.58 & 77.52 & 13.63 & 16.31 \\
\hline \multirow[t]{2}{*}{ Towel Dispenser } & 19 & 37 & 51.35 & 35.89 & 66.55 & 15.20 & 15.46 \\
\hline & 131 & 245 & 53.47 & & & & \\
\hline \multicolumn{8}{|c|}{ Both Hospitals Aggregate Phase 3} \\
\hline Bedroom Light Switch & 5 & 37 & 13.51 & 5.44 & 28.45 & 14.94 & 8.07 \\
\hline Door Handle & 18 & 37 & 48.65 & 33.45 & 64.11 & 15.46 & 15.20 \\
\hline Soap Dispenser & 6 & 37 & 16.22 & 7.27 & 31.52 & 15.30 & 8.95 \\
\hline Grab Bar & 21 & 31 & 67.74 & 50.03 & 81.54 & 13.80 & 17.71 \\
\hline Flush Button & 18 & 30 & 60.00 & 42.29 & 75.44 & 15.44 & 17.71 \\
\hline Tap Handle & 16 & 37 & 43.24 & 28.66 & 59.1 & 15.86 & 14.58 \\
\hline \multirow[t]{2}{*}{ Towel Dispenser } & 17 & 37 & 45.95 & 31.03 & 61.62 & 15.67 & 14.92 \\
\hline & 101 & 246 & 41.06 & 35.09 & 47.3 & 6.24 & 5.97 \\
\hline
\end{tabular}


illustrated that education alone will be insufficient to obtain and sustain on-going improvement in cleaning thoroughness and it has compelled us to invest further in our relationship with EVS leadership and staff.

Accounting for less than $5 \%$ of all acute care inpatient beds, the sample of rooms studied in this project was small. Our study period was short and due to the 48-h periods between marking and reading and the frequent moving of patients and moveable furniture and equipment, we necessarily limited marking to those HTOs permanently affixed to rooms. As well, the thoroughness of cleaning was not independently assessed as PI both collected and analysed data. Given the prestudy agreement made between the PIs regarding continuity and standardisation of marker placement and determination of what constituted and distinguished a 'clean' from 'not clean' HTO, we are confident that our results are reliable. In our facility, infection control does not have programmatic responsibility or oversight for EVS staff. Our work around cleaning continues to be driven by a willingness to work collaboratively with EVS staff for improvement. We see no reason, benefit or value in reporting inaccurate results. The involvement of PIs as assessors is consistent with the methodology used in most similar international reports related to cleaning thoroughness.

These design and methodological issues may limit the generalisability of our findings, however the international expert who served as a co-investigator on our work, also advised that accurate analysis of cleaning thoroughness is possible with few rooms and over short time periods. ${ }^{34}$ Our phase 1 and 2 overall results were similar to phase 1 and 2 rates reported in Carling's seminal work, ${ }^{34}$ suggesting that our sample size and study period may be sufficient to give an accurate indication of typical cleaning thoroughness in our District.

The similarity of our baseline findings to those reported internationally was paradoxically both alarming and reassuring. ${ }^{34,38}$ From a benchmarking perspective we were slightly reassured by the similarity between our baseline compliance levels and those reported internationally. They confirmed the importance of the project, assisted us with EVS leadership engagement and coupled, with images of potentially pathogenic organisms recovered from several of the HTOs swabbed in phase I, provided compelling data and material for inclusion in the EVS education we provided to all EVS staff between phases 1and 2.

The observed variation between cleaning thoroughness for individual HTOs is difficult to explain, as is the significant improvement in only one HTO, towel dispensers, in phase 3 compared with phase 1 . Based on some unprompted feedback provided by EVS in the postphase 1education sessions at both hospitals, we suspect that our EVS staff, like others described in the literature, are reluctant to touch and clean electrical equipment. ${ }^{39}$ This may explain why overall light switches were the least thoroughly cleaned HTO. Given the light switch's status as a 'medium-touch' surface ${ }^{40}$ it is important that our future education includes detailed instruction about the importance of its cleaning. In our understanding standardised District-wide EVS training, cleaning products, and current recommendations included in written and online cleaning procedures did not account for the variation between cleaning thoroughness of individual HTOs and between the two hospitals. Further, we intend to perform thorough analysis of our EVS survey to better understand their work, training and education experiences as well as their current perceptions regarding the frequency with which specific HTOs should be cleaned and the parties responsible for such cleaning. In the interim, ad hoc discussions initiated by EVS staff during and subsequent to the education sessions have been illuminating and helped us to better understand commonly held misperceptions which may be impacting the delivery and frequency of cleaning in our District. They have informed the current stage of our project which has included production of a suite of short training films for all EVS staff. We intend to publish a detailed report of the EVS survey.

EVS leadership and staff were surprised and disappointed by the results of each phase and the study overall. However, individual EVS staff have subsequently approached the infection control department and requested that their specific ward and rooms be included as future sites for HTO marking and covert assessment. During provision of the phase 1 results and before the phase 2 assessment, EVS staff generally embraced the importance of their unique role in HAI prevention and appeared keen to know more about the individual and collective quality of their work and specifically how they could improve cleaning compliance. We suspect that this willingness is reflected in the improvements in both hospitals between phase 1and 2. The lack of sustained improvement between phases 2 and 3 is telling. It likely demonstrates that once-off feedback of data and education without structured, formal and regular interactions between all EVS staff and infection control is insufficient to maintain initial short-term gains. Regardless, along with EVS leadership, the Infection Control Committee and hospital administration, we remain hopeful. We are inspired to identify and deliver corrective strategies including EVS staff role clarification and standardised EVS staff education and practice. Given the imminent relocation of our larger facility in late 2012 to a new, purpose-built hospital with at least 300 more beds and an enhanced range of inpatient and outpatient services, it is crucial that we initiate training and improvements now so as to ultimately achieve workable solutions and sustained high-quality thoroughness of cleaning before the move. Internally we are committed to achieving and maintaining a $90 \%$ or better thoroughness of daily HTO cleaning before and after the relocation.

Surfaces of the HTOs we studied comprised a variety of compositions (plastic, metal, Perspex) and textures (smooth, shiny, flat and raised). By chance they enabled us to confirm 
the FM's suitability for the wide range of HTO surfaces typically found in Australian hospitals. We were unable to quantify or account for patient, visitor or healthcare worker actions that may have inadvertently removed the FM from surfaces. If this did occur it may have resulted in an over estimation of cleaning thoroughness by including marks removed entirely by contact other than cleaning by EVS staff. In a few rooms during the study we noted that used patient towels, which were often damp, or patient clothing were hanging over the grab bar and obscuring the central location where the FM marking was applied.

Other experts suggest that cleaners may be reluctant to touch and move patient's belongings during cleaning, however our study design prevented us from identifying why some HTOs were cleaned while others were not and the extent to which such reluctance is common. ${ }^{39}$ We were unable to fully explain Hospital B's consistently higher performance compared with Hospital A, however at the time we conducted the study, the four Hospital B wards had been accepting patients for less than 4 weeks. Clinical and EVS staff were settling into their wards and verbally displayed pride in their new surroundings and a will to keep those new wards clean and tidy. We are hopeful Hospital A staff will exhibit a similar desire as they relocate to the new facility in late 2012.

There were several unanticipated but common observations investigators noted during the application and mark reading in both hospitals. These included questioning from several inpatients in targeted rooms about what we were doing. After explanation, several inpatients commended us on the initiative. Few investigators have reported strategies for engaging patients as advocates and monitors of cleaning thoroughness. The interest expressed by patients compels us to think more about how patient involvement and self-advocacy could be implemented.

Given the interest of EVS leadership and staff in this process and the case for using FM, we will provide additional training to selected EVS staff so that in future they can regularly and routinely initiate peer and selfassessment of HTO cleaning thoroughness. We predict that careful, supported transfer of responsibility for measuring cleaning thoroughness to EVS staff will increase their buy-in and ultimately improve their performance. However, we are keen to ensure that reports remain accurate, reliable and valid and so it is likely that occasionally the infection control team will undertake both overt and covert observations of cleaning effectiveness. In the event of outbreaks associated with environmental pathogens we expect that the infection control team may use the FM to undertake more focussed ad hoc assessments of cleaning thoroughness in specific wards or units or on specific pieces of equipment.

There are several opportunities to further our work, these include:
- Regular feedback to quality assurance or patient safety staff and hospital leadership through inclusion of cleaning thoroughness data on our monthly infection control 'dashboard';

- Expansion of ward or unit locations and HTOs for consideration of FM use;

- Education of more staffincluding ward-based and EVS staff so that they are competent and independently capable in applying, reading and interpreting FM marks to monitor cleaning thoroughness;

- Regular feedback of the FM results to clinical staff so as to reinforce previous messages about the risk of hand contamination from contaminated environmental surfaces and the importance of hand hygiene, ${ }^{6}$

- More detailed investigation of the impediments to cleaning thoroughness and development and implementation of corrective measures.

Our work confirmed that there are substantial opportunities to measure, better understand and improve the level of cleaning being performed in hospitals. Timely provision of feedback can improve the thoroughness of hospital cleaning. Without on-going, long-term feedback and education these improvements may not be sustained.

Infection control staff face increasing complex microbiological and organisational challenges. Monitoring and reporting of cleaning thoroughness should become a routine part of the infection control professionals' suite of strategies to reduce the organisational HAI risk and burden. In Australia we urgently need to incorporate the use of proven tools to assess thoroughness of cleaning of HTOs and other environmental surfaces in our routine work.

At GCHSD our intention was never to measure associations between cleaning, other process measures, or interventions and infection rates, but rather simply to better understand how thoroughly HTO surfaces were being cleaned across our District. We are well aware that internationally there are alternative commercial products and systems for assessing thoroughness of cleaning however given the current unresolved debate surrounding consistent outstanding performance of any single system and the relative safety, low cost, ease of use and ready availability of FM at GCHSD we are satisfied that it makes an extremely useful addition to our program and we look forward to its on-going use as we strive towards $>90 \%$ thoroughness of cleaning across our entire District.

\section{Conflicts of interest}

GCDHS project participants report no conflict of interest. Dr Carling holds a patent for DAZO ${ }^{\circledR}$ and has a financial consultancy relationship with ECOLAB, USA. ECOLAB Australia and ECOLAB USA supported this study in part by providing necessary supplies of DAZO ${ }^{\circledR}$ and black light technology free of charge. ECOLAB Australia and ECOLAB USA had no role in the selection of, study content, methods, analysis or feedback of results. 


\section{References}

1. Weber DJ, Rutala WA, Miller MB, Huslage K, Sickbert-Bennett E. Role of hospital surfaces in the transmission of emerging health careassociated pathogens: norovirus, Clostridium difficile, and Acinetobacter species. Am J Infect Control 2010; 38(5): S25-33. doi:10.1016/j.ajic.2010.04.196

2. Po JL, Carling PC. The need for additional investigation of room decontamination processes. Infect Control Hosp Epidemiol 2010; 31 (7): 776-7. doi:10.1086/653819

3. Carling PC, Bartley JM. Evaluating hygienic cleaning in health care settings: what you do not know can harm your patients. Am J Infect Control 2010; 38(5): S41-50. doi:10.1016/j.ajic.2010.03.004

4. Dumford DM III, Nerandzic MM, Eckstein BC, Donskey CJ. What is on that keyboard? Detecting hidden environmental reservoirs of Clostridium difficile during an outbreak associated with North American pulsed-field gel electrophoresis type 1 strains. Am J Infect Control 2009; 37(1): 15-9. doi:10.1016/j.ajic.2008.07.009

5. Dancer SJ. The role of environmental cleaning in the control of hospital-acquired infection. J Hosp Infect 2009; 73(4): 378-85. doi:10.1016/j.jhin.2009.03.030

6. Hayden MK, Blom DW, Lyle EA, Moore CG, Weinstein RA. Risk of hand or glove contamination after contact with patients colonized with vancomycin-resistant enterococcus or the colonized patients' environment. Infect Control Hosp Epidemiol 2008; 29(2): 149-54. doi:10.1086/524331

7. Sattar S, Tetro JA. Environmental Survival and Microbicide Inactivation of Some Currently Significant Nosocomial Pathogens. In Rutala WA, ed. Disinfection, Sterilization and Antisepsis. Washington: APIC; 2007.

8. Sattar S, Springthorpe VS. Disinfection of Environmental Surfaces to Interrupt the Spread of Nosocomial Pathogens: a Critical Look at Use Patterns and Expectations. In Rutala WA, ed. Disinfection, Sterilization and Antisepsis. Washington: APIC; 2007.

9. Rutala WA, Weber DJ. Surface Disinfection: new Processes and Products. In Rutala WA, ed. Disinfection, Sterilization and Antisepsis. Washington: APIC; 2007.

10. Dettenkofer M, Spencer RC. Importance of environmental decontamination - a critical view. J Hosp Infect. 2007; 65(Supplement 2): 55-57. doi:10.1016/S0195-6701(07)60016-4

11. Boyce JM. Environmental contamination makes an important contribution to hospital infection. J Hosp Infect. 2007; 65(Supplement 2): 50-54. doi:10.1016/S0195-6701(07)60015-2

12. Sexton T, Clarke P, O'Neill E, Dillane T, Humphreys $H$. Environmental reservoirs of methicillin-resistant Staphylococcus aureus in isolation rooms: correlation with patient isolates and implications for hospital hygiene. J Hosp Infect. 2006; 62(2): 187-194. doi:10.1016/j.jhin.2005.07.017

13. Huang SS, Datta R, Platt R. Risk of acquiring antibiotic-resistant bacteria from prior room occupants. Arch Intern Med 2006; 166(18): 1945-51. doi:10.1001/archinte.166.18.1945

14. French GL, Otter JA, Shannon KP, Adams NMT, Watling D, Parks MJ. Tackling contamination of the hospital environment by methicillinresistant Staphylococcus aureus (MRSA): a comparison between conventional terminal cleaning and hydrogen peroxide vapour decontamination. J Hosp Infect. 2004; 57(1): 31-37. doi:10.1016/j. jhin.2004.03.006

15. Denton M, Wilcox MH, Parnell $\mathrm{P}$, et al. Role of environmental cleaning in controlling an outbreak of Acinetobacter baumannii on a neurosurgical intensive care unit. Journal of Hospital Infection. 2004; 56(2): 106-110. doi:10.1016/j.jhin.2003.10.017

16. Barker J, Vipond IB, Bloomfield SF. Effects of cleaning and disinfection in reducing the spread of Norovirus contamination via environmental surfaces. J Hosp Infect. 2004; 58(1): 42-49. doi:10.1016/j.jhin.2004.04.021
17. Weber DJ, Rutala WA. The role of the environment in transmission of Clostridium difficile infection in healthcare facilities. Infect Control Hosp Epidemiol 2011; 32(3): 207-9. doi:10.1086/658670

18. Stiefel U, Cadnum JL, Eckstein BC, Guerrero DM, Tima MA, Donskey CJ. Contamination of hands with methicillin-resistant Staphylococcus aureus after contact with environmental surfaces and after contact with the skin of colonized patients. Infect Control Hosp Epidemiol 2011; 32(2): 185-7. doi:10.1086/657944

19. Chen LF, Falagas ME, Peleg AY. Facing the rising tide of multidrug resistant Gram-negative pathogens. Healthc Infect 2011; 16(1): 1-5.

20. Conway R, Bunt S, Mathias E, Said H. The Norovirus experience: An exercise in outbreak management at a tertiary referral hospital. Healthc Infect 2005; 10(3): 95-102. doi:10.1071/HI05095

21. Stuart RL, Marshall C, McLaws M-L, Boardman C, Russon PL, Harrington G, et al. ASID/AICA position statement-Infection control guidelines for patients with Clostridium difficile infection in healthcare settings. Healthc Infect 2011; 16(1): 33-9.

22. Van Gessel H, Riley T, McGregor A. Clostridium difficile infection: an update for infection control practitioners. Healthc Infect 2009; 14(3): 115-8. doi:10.1071/HI09105

23. Pearman JW, Peterson AM, Lee RC, Orrell CT, Perry P, Kosaras FP, et al. The epidemiology of a large single-strain outbreak of vancomycin-resistant Enterococcus faecium vanB in an Australian teaching hospital. Healthc Infect 2003; 8(3): 77-87. doi:10.1071/ HI03077

24. Mitchell DH, Dempsey KM, Jelfs PJ. Multi-resistant Acinetobacter baumannii: description of an outbreak centred on an intensive care unit in an Australian hospital. Healthc Infect 1999; 4(2): 12-5. doi:10.1071/ HI99212

25. Farmer T, Mitchell C. Australian Infection Control Guidelines: preventing and managing infection in health care. Healthc Infect 2010; 15(4): 101-3. doi:10.1071/HI10034

26. Dendle C, Martin RD, Cameron DR, Grabsch EA, Mayall BC, Grayson ML, et al. Staphylococcus aureus bacteraemia as a quality indicator for hospital infection control. Med J Aust 2009; 191(7): 389-92.

27. Grayson ML, Russo PL. The national hand hygiene initiative. Med $J$ Aust 2009; 191(8): 420-1.

28. Gillespie EE, Scott C, Wilson J, Stuart R. Pilot study to measure cleaning effectiveness in health care. Am J Infect Control 2011; (in press)

29. Stuart RL, Marshall C. Clostridium difficile infection: a new threat on our doorstep. Med J Aust 2011; 194(7): 331-2.

30. Collignon PJ, Grayson ML, Johnson PD. Methicillin-resistant Staphylococcus aureus in hospitals: time for a culture change. Med $J$ Aust 2007; 187(1): 4-5.

31. Griffith CJ, Obee P, Cooper RA, Burton NF, Lewis M. The effectiveness of existing and modified cleaning regimens in a Welsh hospital. J Hosp Infect 2007; 66(4): 352-9. doi:10.1016/j. jhin.2007.05.016

32. Griffith CJ, Obee P, Cooper RA, Burton NF, Lewis M. The effectiveness of existing and modified cleaning regimens in a Welsh hospital. J Hosp Infect 2007; 66(4): 352-59. doi:10.1016/j. jhin.2007.05.016

33. Carling PC, Von Beheren S, Kim P, Woods C. Intensive care unit environmental cleaning: an evaluation in sixteen hospitals using a novel assessment tool. J Hosp Infect 2008; 68(1): 39-44. doi:10.1016/ j.jhin.2007.09.015

34. Carling PC, Parry MM, Rupp ME, Po JL, Dick B, Von Beheren S. Improving cleaning of the environment surrounding patients in 36 acute care hospitals. Infect Control Hosp Epidemiol 2008; 29(11): 1035-41. doi:10.1086/591940 
35. Carling PC, Parry MF, Von Beheren SM. Identifying opportunities to enhance environmental cleaning in 23 acute care hospitals. Infect Control Hosp Epidemiol 2008; 29(1): 1-7. doi:10.1086/524329

36. Carling PC. Evaluating the thoroughness of environmental cleaning in hospitals. J Hosp Infect 2008; 68(3): 273-4. doi:10.1016/j. jhin.2007.10.023

37. Clinical and Laboratory Standards Institute. Performance Standards for Antimicrobial Disk Susceptibility Tests; Approved. StandardTenth Edition. 2009;29(1).

38. Ragan K, Khan A, Zeynalova N, McKernan P, Baser K, Muller MP. Use of audit and feedback with fluorescent targeting to achieve rapid improvments in room cleaning in the intensive care unit and ward settings. [Corrected Proof.] Am J Infect Control. (in press) doi:10.1016/ j.ajic.2011.04.003
39. Dumigan DG, Boyce JM, Havill NL, Golebiewski M, Balogun O, Rizvani R. Who is really caring for your environment of care? Developing standardized cleaning procedures and effective monitoring techniques. Am J Infect Control 2010; 38(5): 387-92. doi:10.1016/j.ajic.2009.07.005

40. Huslage K, Rutala WA, Sickbert-Bennett E, Weber DJ. A quantitative approach to defining "high-touch" surfaces in hospitals. Infect Control Hosp Epidemiol 2010; 31(8): 850-3. doi:10.1086/655016 
AUTHOR QUERIES

1. Please provide degree and affiliation for Orr

2. Check all affiliations and confirm they are correct

3. Au: Please check the Refs. 31 and 32 repeated twice 